4. Wendorf KA, Kay M, Baliga C, et al. Endoscopic retrograde cholangiopancreatography-associated AmpC Escherichia coli outbreak. Infect Control Hosp Epidemiol 2015;36:634-642.

5. Aumeran C, Poincloux L, Souweine B, et al. Multidrug-resistant Klebsiella pneumoniae outbreak after endoscopic retrograde cholangiopancreatography. Endoscopy 2010;42:895-899.

6. Framework for action on interprofessional education and collaborative practice. World Health Organization website. http:// www.who.int/hrh/resources/framework_action/en/ Published 2010. Accessed April 10, 2018.

7. Walz JM, Ellison RT, Mack DA, et al. The bundle "Plus": the effect of a multidisciplinary team approach to eradicate centralline-associated bloodstream infections. Anesth Analg 2015;120: 868-876.

8. Gillespie BM, Kang E, Roberts S, et al. Reducing the risk of surgical site infection using a multidisciplinary approach: an integrative review. J Multidiscip Healthc 2015;8:473-487.

9. Miller R, Simmons S, Dale C, Stachowiak J, Stibich M. Utilization and impact of a pulsed-xenon ultraviolet room disinfection system and multidisciplinary care team on clostridium difficile in a longterm acute care facility. Am J of Infect Control 2015;43:1350-1353.

\section{Do Periarticular Joint Injections Present an Increase in Infection Risk?}

To the Editor-Joint replacements are projected to be the most common elective surgical procedures in the coming decade. ${ }^{1,2}$ Effective management of postoperative pain associated with joint replacement surgery improves surgical outcomes by increasing postoperative mobility and reducing the duration of hospitalization. ${ }^{3}$ Many surgeons have recently incorporated local analgesia into pain management regimens for patients undergoing joint replacement surgery. ${ }^{4,5}$ The benefits of using periarticular injections (PAIs) include decreasing opioid consumption and its associated side effects, facilitating earlier mobilization, and decreasing hospital length of stay. ${ }^{6}$ Most PAI techniques involve infiltration of a high-volume, long-acting local anesthetic solution into the surgical incision and surrounding tissues prior to skin closure. A wide variety of medications is used in these injections. ${ }^{7}$ However, based on our hospitals' experiences, these injections frequently include epinephrine, ketorolac, opioids, or steroids.

The Duke Infection Control Outreach Network (DICON) recently identified the use of PAIs as a potential risk for infection during 4 investigations of clusters of SSIs associated with total hip replacements (THRs) and total knee replacements (TKRs). We surveyed hospitals within our network to gather more information about PAI practices. Of 42 hospitals, 20 (48\%) participated in the survey. Of these 20 hospitals, $16(80 \%)$ reported routine use of PAIs in patients undergoing joint replacement surgery. All 16 hospitals used PAIs in patients undergoing TKRs; 14 hospitals also used PAIs in patients undergoing THRs. In 5 hospitals, PAIs included a combination of bupivacaine, morphine, and ketorolac. The remaining 11 hospitals used highly variable combinations of medications. In 12 hospitals, PAIs were prepared in the operating room (OR), while in the other 4 hospitals, PAIs were prepared in the pharmacy. Among the 4 clusters of SSIs we investigated, all prepared PAIs in the OR.

The current literature regarding the relative infection risk associated with periarticular injection use compared to other modalities of pain control is inconclusive. Marques et $\mathrm{al}^{8}$ performed a systemic review and meta-analysis of 2,348 patients undergoing joint replacement surgery (909 THR, 1,439 TKR). ${ }^{8}$ Only eight cases of deep infection requiring surgical debridement or revision occurred in the cohort (overall infection risk, $0.34 \%$ ). In patients with THRs, four deep infections occurred in patients who received PAIs, and one deep infection occurred in the control group (Peto OR $3.47 ; 95 \%$ CI $0.58,20.81 ; P=0.17$ ). In patients with TKRs, two deep infections occurred in patients who received PAIs compared to one deep infection that occurred in the control group (Peto OR 1.85; 95\% CI 0.19, 17.83; $P=0.59$ ). Thus, the increased number of infections in the PAI groups was not statistically significant. The six patients with deep infection after PAIs received their PAI through a postsurgical catheter that remained in place following surgery.

Jiang et $\mathrm{al}^{9}$ performed a meta-analysis that included an analysis of wound complication rates following TKA and THA in which PAIs were administered. This study showed no statistically significant difference in wound complication rates for either surgery type among patients who received PAIs compared to those who did not. Wang et $\mathrm{al}^{10}$ performed a meta-analysis of ten studies that compared pain control in patients who received PAIs after TKA and patients who received a nerve block. Of these studies, 3 reported that wound complications were not significantly increased in the PAI group (odds ratio, $1.57 ; 95 \% \mathrm{CI}, 0.40-6.16 ; P=.52)$. Although the preceding studies suggest that rates of infection-related complications in patients undergoing PAIs are low, most of the previous studies were underpowered or included an inadequate duration of follow-up to detect an association between PAI and SSI risk.

From a theoretical and practical perspective, the use of PAIs has numerous potential risks for the introduction of bacteria into the joint space or incisional tissues. For example, PAIs are usually compounded in the OR without the use of a sterile hood. Furthermore, OR personnel who lack formal training in drug compounding are typically responsible for preparing these injections. Bacteria may also be introduced through the skin at the time of drug injection, especially if 
external catheters are used to infuse analgesic medications. Finally, many surgeons include corticosteroids as an adjuvant medication in these injections, which may increase the risk of infection.

Our survey of community hospitals revealed widespread use of PAIs without standardization of drug composition, compounding techniques, and method of administration. We were unable to prove that PAIs were responsible for the 4 clusters of SSI reported above. These outbreaks stopped after a number of interventions were undertaken, including standardization of compounding techniques or discontinuation of PAIs. The use of PAIs and the associated risks of infection may lead to sporadic occurrences or clusters of SSIs at other hospitals. However, it is difficult to determine the cause of small clusters of SSIs, and publication bias may limit evaluation of PAI safety. Therefore, hospital epidemiologists and surgeons should be aware of these potential risks, especially if an increase in rates of SSI following joint replacement surgeries occurs at their institution.

\section{ACKNOWLEDGMENTS}

Financial support: No financial support was provided relevant to this article.

Potential conflicts of interest: All authors report no conflicts of interest relevant to this article.

\section{Jessica Seidelman, MD; ${ }^{1,2}$ Arthur W. Baker, MD, MPH; ${ }^{1,2}$ Deverick J. Anderson, MD, MPH; ${ }^{1,2}$ Daniel J. Sexton, MD; ${ }^{1,2}$ Sarah S. Lewis, MD, MPH ${ }^{1,2}$}

\footnotetext{
Affiliations: 1. Duke Center for Antimicrobial Stewardship and Infection Prevention, Durham, North Carolina; 2. Division of Infectious Diseases, Duke University School of Medicine, Durham, North Carolina.
}

Address correspondence to Jessica Seidelman, 310 Trent Drive, Hanes House Room 181, Durham, NC 27710 (jessica.seidelman@duke.edu).
Infect Control Hosp Epidemiol 2018;39:889-891

(C) 2018 by The Society for Healthcare Epidemiology of America. All rights reserved. 0899-823X/2018/3907-0025. DOI: 10.1017/ice.2018.80

\section{REFERENCES}

1. Cram P, Lu X, Kates SL, Singh JA, Li Y, Wolf BR. Total knee arthroplasty volume, utilization, and outcomes among Medicare beneficiaries, 1991-2010. JAMA 2012;308:1227-1236.

2. Kurtz SM, Lau E, Ong K, Zhao K, Kelly M, Bozic KJ. Future young patient demand for primary and revision joint replacement: national projections from 2010 to 2030. Clin Orthop Relat Res 2009;467:2606-2612.

3. Kehlet H, Holte K. Effect of postoperative analgesia on surgical outcome. Br J Anaesth 2001;87:62-72.

4. Kerr DR, Kohan L. Local infiltration analgesia: a technique for the control of acute postoperative pain following knee and hip surgery: a case study of 325 patients. Acta Orthop 2008;79:174-183.

5. Yin JB, Cui GB, Mi MS, et al. Local infiltration analgesia for postoperative pain after hip arthroplasty: a systematic review and meta-analysis. J Pain 2014;15:781-799.

6. Russo MW, Parks NL, Hamilton WG. Perioperative pain management and anesthesia: a critical component to rapid recovery total joint arthroplasty. Orthop Clin North Am 2017;48:401-405.

7. Kelley TC, Adams MJ, Mulliken BD, Dalury DF. Efficacy of multimodal perioperative analgesia protocol with periarticular medication injection in total knee arthroplasty: a randomized, double-blinded study. J Arthroplasty 2013;28:1274-1277.

8. Marques EM, Jones HE, Elvers KT, Pyke M, Blom AW, Beswick $\mathrm{AD}$. Local anaesthetic infiltration for peri-operative pain control in total hip and knee replacement: systematic review and meta-analyses of short- and long-term effectiveness. BMC Musculoskelet Disord 2014;15:220.

9. Jiang J, Teng Y, Fan Z, Khan MS, Cui Z, Xia Y. The efficacy of periarticular multimodal drug injection for postoperative pain management in total knee or hip arthroplasty. J Arthroplasty 2013;28:1882-1887.

10. Wang C, Cai XZ, Yan SG. Comparison of periarticular multimodal drug injection and femoral nerve block for postoperative pain management in total knee arthroplasty: a systematic review and meta-analysis. J Arthroplasty 2015;30:1281-1286. 